\title{
IoT Enabled Wearable Camera for Emerging Application World
}

\author{
Krishna Patel \\ U and P. U. Patel Department of Computer Engineering, \\ CSPIT, CHARUSAT, Changa, Gujarat, India \\ Email: nkrishnapatel@gmail.com \\ Nikita Bhatt \\ U and P. U. Patel Department of Computer Engineering, \\ CSPIT, CHARUSAT, Changa, Gujarat , India \\ Email: nikitabhatt.ce@charusat.ac.in
}

ABSTRACT-

IoT Enabled Wearable Camera is used to make world's smallest wearable camera that can capture video clips. This hardware is a wearable camera device, which users can mount on their sunglasses, goggles and helmets using magnets already available in the camera. This Camera gives better resolution of video. And to store these videos and to charge cameras we have one docking station. The docking station that can charge camera and also transferred video wirelessly. With the help of wifi , stored video can be directed uploaded On cloud. With the use of mobile application we can directly take audio and video from cloud. The main aim is to develop smallest wearable camera that have most capabilities that can fulfill our daily basis need.

Keywords - Wearable camera , IoT , Cloud , Docking station

\section{INTRODUCTION}

“Internet of things refers to the ever-growing network of physical objects that feature an IP address for internet connectivity, and the communication that occurs between these objects and other internet-enabled devices and systems. Internet of things is an ecosystem of connected physical objects that are accessible through the internet. IoT enabled wearable camera that magnetically mounts to sunglass, goggles, helmets and other items. IoT enabled wearable camera solution consists of two modules, Camera and docking station. Camera records video and audio data to storage. When camera is present into docking station, data recorded in camera is transferred to storage of docking station for preview to mobile device via wireless interfaceWi-Fi/BT. Video resolution of recording at either 720p@60 fps or 1080p@30fps.To transfer a video to phone, we can use both Bluetooth and Wi-Fi. Wi-Fi is used for high speed transfer. This smallest camera is used to capture video. Wear camera around neck or on figure as a ring.Use application to share videos and audio. The main aim is to develop this camera, which is used to capture some special moments where the camera cannot be handy. For example, capturing a video while climbing a mountain is possible with this camera. Using Wi-Fi access data from cloud. With the use of mobile application, store the videos to our mobile device and then share these videos to other users easily. Wearable camera is used to capture video and some special moments where handheld camera cannot be used. For example, video capturing while surfing or dirt biking. The storage capacity of wireless camera is $4 \mathrm{~GB}$. Gateways are responsible for routing the processed data and send it to proper location. With the help of wifi, stored videos will be directly uploaded on the cloud. In this automated generation, to make users work easy, whenever the camera will be connected to the wifi hotspot it will automatically upload data on cloud. With the use of mobile application we can directly take video and audio from cloud. Also transfer video and audio to other user with the use of mobile application. Cloud includes User management, Data storage and Web management. User management describes administrators to manage user access to different IT resources like systems, devices, applications, storage and more. IT admin create, manage and delete users on cloud. Logging users, managing users, account and user modification, handling the termination process, communication with the users all things includes in user management. Cloud storage on which data is stored on server can be accessed from internet. Web management provides a link through which we can directly take video/audio from cloud.IoT enabled wearable camera that magnetically mounts to sunglass, goggles, helmets clothes and other items. IoT enabled wearable camera solution consists of two modules, Camera and Docking Station. Camera records video and audio data to storage memory. Docking station used to charge camera and transfer video clips from camera to mobile devices. Using mobile application video clips can be shared on social media like (FB, Twitter, etc). Also, mobile application is used to configure and control camera.

\section{RELATED WORK}

In [1], the purpose of this product is violation and rape cases victim's cases, which is increasing now a days. Women and children are not safe. Now a days women are suffering more rape cases in the society so for that intelligent hidden camera is been developed. So that moments is captured with the help of that it should decide 
whether the victim is in danger or not. And according to that alert system and locating tracking chip is also been added with the help of IoT system, security is been supported. In this while capturing the video it will directly transmit it to the cloud. There is any danger it will directly contact to the authorized contact list. Hand gestures and facial reorganization method is been used to detect. The proposed system is developer for security purpose of women and children for their security purpose. The planned camera system is supported with internet to connect the device with our home and authorized officers. Camera system recording audio/video and storing video stream. Then transmittal video streaming into cloud platform. With the utilization of net good phone simply take video/audio from cloud platform. The hardware 0.5 consists of processor and a camera system, the position of the device is tracked associated degree AN alert message is transmitted with the involvement of GSM and GPS module.

In [2], "the common observe of manual synchronization of body-worn, and video cameras is for integration into everyday observe for applications like real-world behavior analysis. We've a bent to increase associate existing technique for automatic synchronize and live its performance throughout a true experimental setting. Special visual communication, captured by a camera, are matched with recorded signal exploitation crosscorrelation based time-delay estimation. PCA-based data pre-processing makes the plan of action against direction unsimilarity between the marking visual communication and thus the camera plane." Supported a literature survey we have a tendency to develop and enforced a synchronization technique wherever wears of accelerometers perform bound marker gestures ahead of the camera. The temporal relation technique automatically detects video and measuring instrument information and calculates normalized cross-correlation values. We have a tendency to have an interest during good and simple to use automated synchronism technique for the subsequent usecase. The overall set up for either manual or automatic synchronization in capturing certain specific activities sequences by all modalities involved.

In [3], "the implementation of style and system technique flow with functions will facilitate the wearers amount of your time face realize, recognition and identification. The formula produce with capturing adequate build video frames from wearable camera followed by interested object realize and detection. We have a tendency to tend to indicate effectiveness and additionally the usefulness of this system by experimental results." The aim of Face Detection Module is to search out the face that the police want. To avoid repeatedly police investigation wrong object and utilize the more resource plenty of efficiently, to implement module is split into 2 steps to implement: Error improvement and face find. Period of time face find is one in all the foremost in style and necessary problems within the domain of laptop vision. Nowadays amount of your time face notice technology has wide used in face access system, flying field safety and intelligent transportation etc. Machine learning high tech is employed to realize higher ends up in object find space. The illustration purpose of read supported machine learning technique is SVM, theorem classifier and many more.

In [4], Startle Cam can be a wearable video camera, computer, and hold system, which allows the camera to be contained via events involving the user. The aim is to capture events and store into system. Startle Cam monitors the wearer's skin physical phenomenon. at intervals the other mode, the camera are usually set to automatically record photos at a gaggle frequency once solely some responses are identify from the user, illustrate that their attention level has born. Startle Cam could be a wearable ADPS with a digital video camera, physiological sensors and a wireless affiliation to the web. Method formula to note the jump, to hold a move buffer of video photos from the photographic equipment, and to manage the outflow of video knowledge to the disc or the web. Pictures from the camera are hold on during a block of memory till the Startle Cam detection formula is triggered. "Startle Cam system be found in of a skin natural phenomenon detector (GSR) that's sampled by associate analog to digital device attached to a wearable laptop. A photographic equipment and digital equipment are also attached to the laptop." Photos are taken by the photographic equipment and detain an awfully buffer in memory. Once the laptop formula identify a jump, the buffer of images is downloaded and translate wirelessly back to the web.

In [5], The intension of the paper was to spot the foremost moral problems connected to the utilization of wearable cameras in analysis. An opposition studies going down in an exceedingly controlled environments, studies within the free captures knowledge from or concerning participates in their everyday surroundings. Wearable camera that take pictures and their activities over periods of your time. Pictures from wearable cameras even have the potential to provide LTM prompts for participants to self-reflect on their activities through interviews and diaries. "The camera could be a tiny device which will be worn round the neck or clipped onto covering. It's a battery lifetime of around ten hours, creating it possible for analysis participants to wear it for many of on a daily basis. The Narrative camera is an excellent smaller camera that takes a still image each thirty seconds and has a calculable battery lifetime of 2 days. Wearable cameras have the potential to assist with a spread of knowledge capture techniques through the power to support reflection and recollection, increase dependability of self-report, and validate different techniques. As results of potential enhancements to knowledge assortment, quality of analysis output could also be improved. This deserves more investigation on the worth to society of those improved ways. Wearable camera use could be a recently rising space of thought for researchers and this paper has tried to focus on potential problems which will be encountered. It's troublesome to provide a comprehensive set of pointers for managing cameras at this early stage of use. Instead ethics committees have to rigorously 
contemplate the justification for the technique given the balance of risks."

In [6], "The economical use of image sensors has been one amongst the highest challenges for laptop vision researchers for a few years. Police work and pursuit objects, video observation, navigation, and lots of different period applications rely on motion estimation for moving camera. During this paper, a period technique for the detection and classification of the motion of a wearable, moving Vision camera is planned. This approach was adoptive to be used with sensible glasses to help folks with field of vision defects. These categories cowl totally different degrees of freedom as well as rotation and translation. The advocate technique classifies the sort of camera motion as static, translation/rotation left, and right, up or down. This classification is vital for object detection and pursuit which will alert the user to the potential hazards outside their field of read. The planned approach has been tested on a true first-person perspective video captured by a wearable camera. The beta results demonstrate that the planned technique classifies the sort of motion with success in period and may be used as a part of low-priced wearable solutions for numerous kinds of vision loss dependable technologies."

\section{LIMITATIONS OF CURRENT WORK}

It additionally also acts as a security system in vital condition. Associate degree and hardware has been developed to gather video from the camera aspect. The video streams are any processed to activate the overall system. The system to boot establishes Associate in nursing web association that allows the system to connect with Smartphone. Reaction of direction modification of horizontal body language on downtown estimation. Automatic alternative of the camera to be used for synchronism will increase the wise value of the way for multi-camera setups. Sometimes the detection module detects wrong objects. And utilize a lot of resources with efficiency. The differential video camera shown here could be a black and white Connect fast Cam, which might capture up to fifteen frames per second of 320 by 240 constituent pictures in 6-bitgray. "One of the most challenges during this field is that the camera evolution itself. As a result of the camera is wearable, its movements random and unpredictable. Therefore, head (or camera) motion detection could be a key step before beginning different video process procedures. Camera motion kind detection part is vital to make your mind up if the camera is moving or not (stationary) and detects the motion kind." The main challenge of IoT Enabled Wearable Camera is size. Because the size of wearable camera is small, it imposes the restrictions on the size of batteries that can be used in camera. Suppose we want to increase capacity of battery, it directly impacts the size of wearable camera. We cannot capture video more than 8 minutes if video resolution is 1080p 30 frames per seconds due to battery. It's poor choice if someone wants to take long time activity such as climbing, hiking and some special long moments. The other open challenge to this device is to control the impact of temperature on battery life as well as performance. Since the size of camera is small, we cannot control the heat generated inside it above certain limit.

\section{EXPERIMENTAL SETUP}

The chapter includes the experiment and simulation done using tools and the results showing the improvement done by modifying the existing approach in terms of changing parameter and function modification which give the better accuracy and performance. WPA_SUPPLICANT is a text file that lists networks and security policies including preshared key. The use of WPA_SUPPLICANT here is to connect mobile and docking station via hotspot.

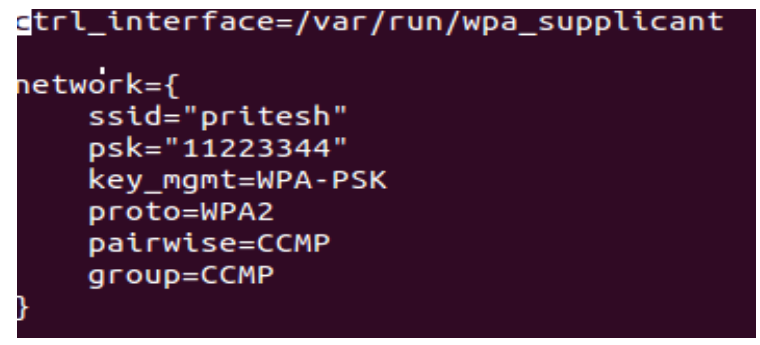

Fig. 1. Wpa_supplicant interface

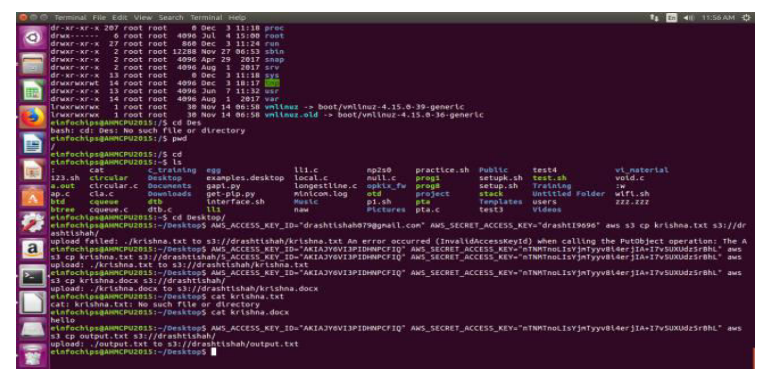

Fig. 2. Command line interface for AWS

The amazon web service command line interface is used to upload text file to $\mathrm{S} 3$ bucket. In this command line interface aws_access_key_id and aws_secret_access_key must be write in command line interface.

\section{SIMULATION RESULTS}

The output of experiment is that whenever will switch on the hotspot from mobile phone and if connect it with system's Wi-Fi. Once it gets connected it will display the IP address of the mobile phone which is connected.

Fig. 3. whenever hotspot connected to system display IP address

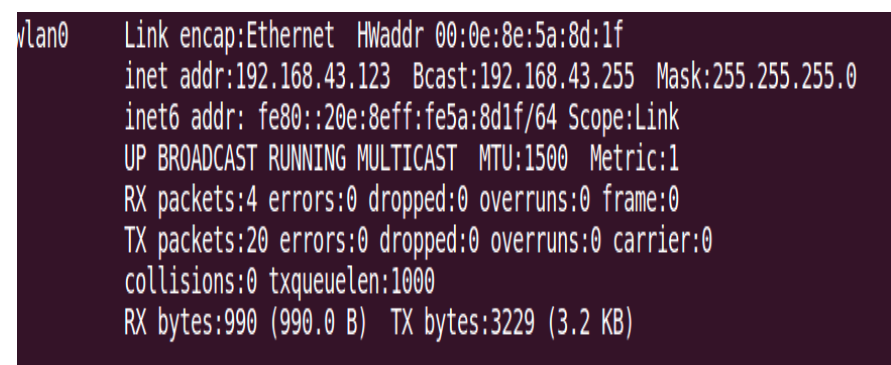

The output of amazon web service command line interface is to check successful upload file on amazon S3. Output.txt file is successful show in AWS S3. 


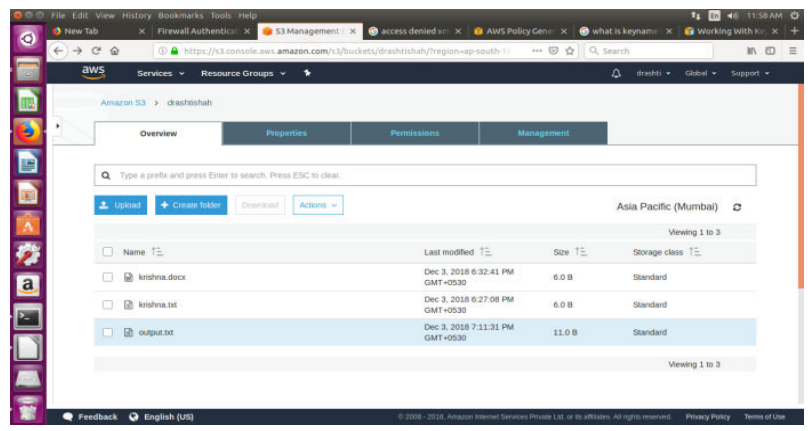

Fig. 4. Successfully uploaded files on aws

To upload all videos on AWS,first check folder is available or not in folder all video files are there.The name of folder is DATA. In DATA folder all files are present like .txt and .$m p 4$ but only .mp4 files are upload on AWS.All files are stored in data.txt then upload data.txt on AWS.Using c++ code first create bucket and then put objects into bucket.All .mp4 files are stored into bucket.

Fig.5. Folder is present or not and folder in files are available or not

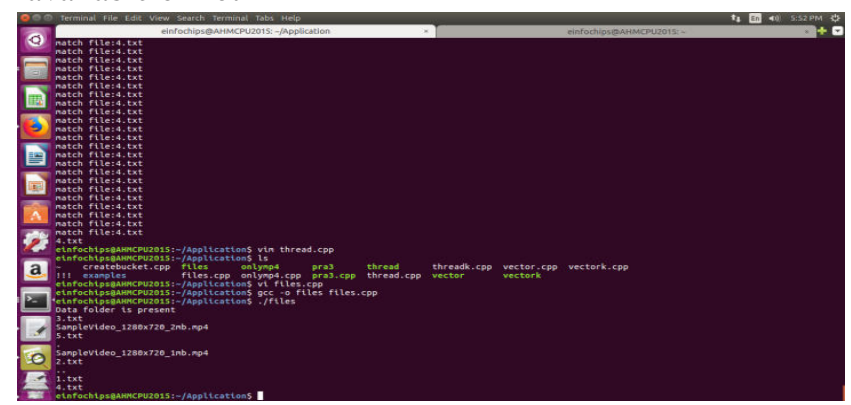

Fig. 6. Show only .mp4 files

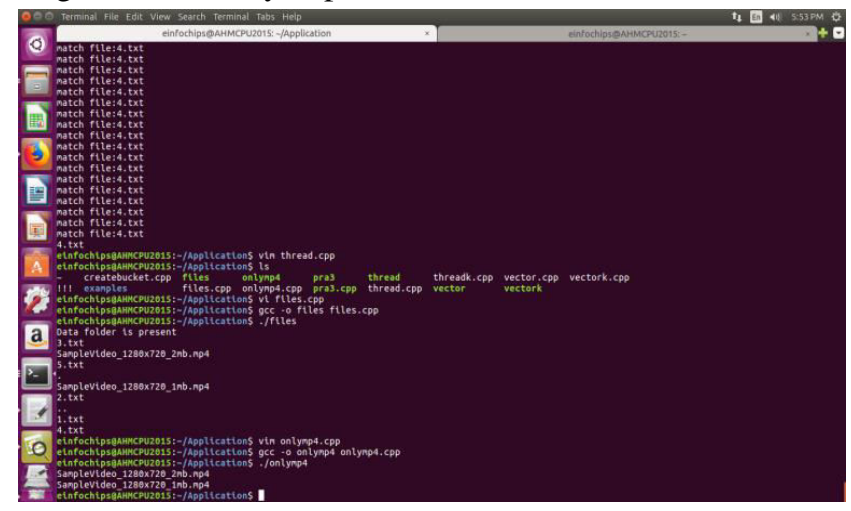

\section{FUTURE WORK}

In future, we can try to implement the design such that we do not require docking station for transferring the videos from camera to mobile phone. Instead of this, the videos are directly transferred from camera to phone using Wi-Fi. For this one can implement such design that Wi-Fi is directly inserted in camera.

\section{CONCLUSION}

The work conferred here is to advance IoT Enabled Wearable Camera that camera is used to capture video and a few special moments where hand-held camera can't be used. With the help of Wi-Fi, stored videos will be directly uploaded on the cloud. In this automated generation, to make users work easy, whenever the camera will be connected to the Wi-Fi hotspot it will automatically upload data on cloud. With the use of mobile application we can directly take video and audio from cloud. Also transfer video and audio to other user with the use of mobile application.

\section{REFERENCES}

1.Han, L., Li, Z., Zhang, H., \& Chen, D. (2010, July). Wearable observation supporting system for face identification based on wearable camera. In Computer Science and Information Technology (ICCSIT), 2010 3rd IEEE International Conference on (Vol. 7, pp. 91-95).

2. Healey, J., \& Picard, R. W. (1998, October). Startlecam: A cybernetic wearable camera. In Wearable Computers, 1998. Digest of Papers. Second International Symposium on (pp. 42-49). IEEE

3.Shipp, V., Skatova, A., Blum, J., \& Brown, M. (2014, May). The ethics of wearable cameras in the wild. In Proceedings of the IEEE 2014 International Symposium on Ethics in Engineering, Science, and Technology (p. 18). IEEE Press

4.Plotz, T., Chen, C., Hammerla, N. Y., \& Abowd, G. D. (2012, June). Automatic Synchronization of Wearable Sensors and Video-Cameras for Ground Truth Annotation-A Practical Approach. In Wearable Computers (ISWC), 2012 16th International Symposium on (pp. 100-103). IEEE.

5.Mann, S. (1997). Smart clothing: The wearable computer and wearcam. Personal Technologies, 1(1), 21-27.

6.Younis, O., Al-Nuaimy, W., Rowe, F., \& Alomari, M. H. (2018, July). Real-time Detection of Wearable Camera Motion Using Optical Flow. In 2018 IEEE Congress on Evolutionary Computation (CEC) (pp. 1-6).

7.S. Mann. Shootingback. the winners of the prix ars electronica1997, $\quad$ Sep $\quad 8-13 \quad 1997$. http://www.rito.com/prix/winners.htm See also,http://wearcomp.org/shootingback.html.

8.S.Mann. Eyeglass mounted wireless video: Computersupported collaboration for photojournalism and everyday use. IEEE ComSoc,pages 144-151, June 1998. Special Issue on Wireless Video.

9.LuHan, Zushu Li, Dongyi Chen "iWearSA: an intelligent Wearable Assistant System for perception of moving object", ICMIT 2007,Proc. SPIE Vol. 6794, pp. 67945M67945M-5

10.G. O'Loughlin, S.J. Cullen, A. McGoldrick, S. O'Connor, R. Blain, S.O'Malley, and G.D. Warrington. “ Using a wearable camera to increase the accuracy of dietary analysis", in American Journal of Preventive Medicine, 2013, 44(3):297-301.

11.L.Miller and J.Toliver , "Implementing a body worn camera",Police executive research forum and United States of America, 2014.

12.Juang C F, Wu J R, Chang C M, et al. "Computer vision-based human body segmentation and posture estimation. Systems,Man and Cybernetics, Part A: Systems and Humans", IEEE Transactions on, 2009,39(1:119-133). 